\title{
AUTHOR INDEX Volume 6, 1996
}

Achour, S., see Bardou

Adachi, M., see Glass

Afraimovich, V.S., Chow, S.-N. \& Shen, W.-X., Hyperbolic homoclinic points of ' $Z^{d}$-actions in lattice dynamical systems

Aguirre, B., see Suárez

Aguirre, L.A., \& Mendes, E.M.A.M., Global nonlinear polynomial models: Structure, term clusters and fixed points

Ahammer, H., see Windisch

Aihara, K., see Tokuda

Albano, A.M., see Storella

Almeida, P., see Markus

Alvarez-Ramirez, J., see Suárez

Andreyev, Yu.V., Dmitriev, A.S., Kuminov, D.A., Chua, L.O. \& Wu, C.W., I-D maps, chaos and neural networks for information processing

Anishchenko, V.S., see Feudel

Antoniou, I., Basios, V. \& Bosco, F., Probabilistic control of chaos: The B-adic Renyi map under control

Arecchi, F.T., see Giaquinta

Arnold, L., Sri Namachchivaya, N. \& Schenk-Hoppe, K.R., Toward an understanding of stochastic Hopf bifurcation: A case study

Aronson, D.G., see Huang

Arrowsmith, D.K., Lansbury, A.N. \& Mondragón C, R.J., Control of the Arnold circle map

Atela, P., \& Hu, J., Commuting polynomials and polynomials with same Julia set

Auger, P., see Bardou

Bai, F.-S., Lord, G.J. \& Spence, A., Numerical computations of connecting orbits in discrete and continuous dynamical systems

Barbé, A., Shift-add correlation patterns of orbits of onedimensional linear cellular automata

Barbé, A.M., Coarse-Graining invariant orbits of one-dimensional $Z_{p}$-linear cellular automata

Bardou, A., Achour, S., Auger, P. \& Chasse, J.-L., Effects of local Ischemia and transient conduction blocks on the induction of cardiac re-entries

Basios, V., see Antoniou

Batlle, C., Fossas, E. \& Olivar, G., Stabilization of periodic orbits in variable structure systems. Application to $D C$-DC power converters

Beckmann, P.E., see Klemm

Belykh, V.N., Homoclinic orbits bifurcations of one- and twodimensional maps

Bersini, H., see Calenbuhr

Bezerianos, A., see Kremmydas

Biktashev, V.N., Holden, A.V. \& Nikolaev, E.V., Spiral wave meander and symmetry of the plane

Birbaumer, N., Lutzenberger, W., Rau, H., Braun, C. \& MayerKress, G., Perception of music and dimensional complexity of brain activity

Blázquez. M., \& Tuma, E., Chaotic behavior of orbits close to a heteroclinic contour

Blum, L., Cucker, F., Shub, M. \& Smale, S., Complexity and real computation: A manifesto

Boccaletti, S., see Giaquinta

Bode, M., see Schütz

Borckmans, P., see Rasmussen

Bosco, F., see Antoniou

Bountis, T., see Kremmydas

Bountis, T.C., see Vrahatis
Bragard, J., Pontes, J. \& Velarde, M.G., Patterns, defects, and evolution of Benard-Marangoni cells

Brailove, A.A., \& Linsay, P.S., An experimental study of a population of relaxation oscillators with a phase-repelling mean-field coupling

Brandt, M.E., \& Chen, G., Feedback control of a quadratic map model of cardiac chaos

Braun, C., see Birbaumer

Brown, R., \& Chua, L.O., From almost periodic to chaotic: The fundamental map

Brown, R., \& Chua, L.O., Clarifying chaos: Examples and counterexamples

Brucoli, M., Carnimeo, L. \& Grassi, G., A method for the synchronization of hyperchaotic circuits

Bunimovich, L.A., Many-dimensional Lorentz cellular automata and Turing machines

Cabrera, J.L., \& de la Rubia, F.J., Analysis of the behavior of a random nonlinear delay discrete equation

Calenbuhr, V., Varela, F.J. \& Bersini, H., Natural tolerance as a function of network connectivity

Campos, J., see Poon

Campos, J., see Poon

Cao, L.-Y., Fang, H.-P., Li, Q.-L. \& Chen, J.-B., Forecasting $b$ values for seismic events

Carcasses, J.-P., see Mira

Carnimeo, L., see Brucoli

Carr, T.W., \& Schwartz, I.B., Subharmonic-Saddle connections as a precursor to chaos in a periodically modulated laser

Casaleggio, A., Corana, A., Ranjan, R. \& Thakor, N.V., Dimensional analysis of the electrical activity in fibrillating isolated hearts

Castellanos, A., see Pérez

Celletti, A., Froeschle, C. \& Lega, E., Determination of the frequency vector in the four-dimensional standard mapping

Chacón, R., see Pérez

Champneys, A.R., Kuznetsov, Yu.A. \& Sandstede, B., A numerical toolbox for homoclinic bifurcation analysis

Chang, H.-C., see Chen

Chapeau-Blondeau, F., \& Godivier, X., Stochastic resonance in nonlinear transmission of spike signals: An exact model and an application to the neuron

Chasse, J.-L., see Bardou

Chen, C.-C., Hwang, C., Wolf, E.E. \& Chang, H.-C., Characterization of chaotic attractors in extended systems

Chen, G., \& Lai, D., Feedback control of Lyapunov exponents for discrete-time dynamical systems

Chen, G., Zhou, J. \& Hsu, S.-B., Linear superposition of chaotic and orderly vibrations on two serially connected strings with a Van der Pol joint

Chen, G., see Moiola

Chen, G., see Brandt

Chen, G.-R., see Xie

Chen, J.-B., see Cao

Chen, S.-L., \& Shaw, S.W., A fast-manifold approach to Melnikov functions for slowly varying oscillators

Chen, Y.-Q., \& Pei, L.-Q., Analysis of the recovered signal in synchronized Chua's communication system

Chen, Y.-Q., \& Pei, L.-Q., Analysis of the recovered signal in synchronized Chua's communication system (Errata)

Chiacchiarini, H.G., see Moiola
1509

2587

715

2153

545

2441

661 
Chin, C.-M., see Nayfeh

Chiou-Tan, F.Y., Magee, K.N., Robinson, L.R., Nelson, M.R., Tuel, S.S., Krouskop, T.A. \& Moss, F., Enhancement of subthreshold sensory nerve action potentials during muscle tension mediated noise

Chow, S.-N., see Afraimovich

Chow, S.-N., Mallet-Paret, J. \& Van Vleck, E.S., Dynamics of lattice differential equations

Christian, M., About two-dimensional piecewise continuous noninvertible maps

Christov, C.l., see Pontes

Chua, L.O., see Brown

Chua, L.O., see Kuznetsov

Chua, L.O., see Nekorkin

Chua, L.O., see $W u$

Chua, L.O., see Crounse

Chua, L.O., see de Castro

Chua, L.O., Yang, T., Zhong, G.-Q. \& Wu, C.W., Adaptive synchronization of Chua's oscillators

Chua, L.O., see Gligoroski

Chua, L.O., see Brown

Chua, L.O., see Yang

Chua, L.O., see Kapitaniak

Chua, L.O., see Wu

Chua, L.O., see Kozlov

Chua, L.O., see Andreyev

Chua, L.O., see Wu

Conley, A.J., Centrifugal destabilization and restabilization of plane shear flows

Corana, A., see Casaleggio

Crounse, K.R., Chua, L.O., Thiran, P. \& Setti, G., Characterization and dynamics of pattern formation in cellular neural networks

Cucker, F., see Blum

Dabke, K.P., see Lipton

Dabke, K.P., see Lipton

Dabke, K.P., see Lipton

Dąbrowski, A., see Ogorzalek

Dąbrowski, W., see Ogorzalek

Dankowicz, H., Analytical expressions for stable and unstable manifolds in higher degree of freedom Hamiltonian systems

Dankowicz, H., Looking for chaos. An extension and alternative to Melnikov's method

Dapra, D., see Windisch

de Castro, M., Pérez-Muñuzuri, V., Marino, I.P., Gómez-Gesteira, M., Chua, L.O. \& Pérez-Villar, V., Interaction of re-entries in coupled parallel fibers

de Castro, M., see Muñuzuri

de la Rubia, F.J., see Cabrera

Deng, B., Exponential expansion with principal eigenvalues

Deng, B., Spiral-plus-saddle attractors and elementary mechanisms for chaos generation

Denis, P.St., see Mar

Desages, A.C., see Moiola

Deutsch, A., Orientation-induced pattern formation: Swarm dynamics in a lattice-gas automaton model

Dewel, G., see Rasmussen

di Bernardo, M., An adaptive approach to the control and synchronization of continuous-time chaotic systems

Diamond, P., \& Pokrovskii, A., Statistical laws for computational collapse of discretized chaotic mappings

Didoné, G., see Pandin

Dimovski, D., see Gligonoski

Ding, M., see Hilborn

Dmitriev, A.S., see Andreyev
Dmitriev, A.S., Panas, A.I. \& Starkov, S.O., Ring oscillating systems and their application to the synthesis of chaos generators

Ebeling, W., Freund, J. \& Rateitschak, K., Entropy and extended memory in discrete chaotic dynamics

Eleonsky, V.M., Korolev, V.G., On coding of series of homoclinic loops

Encinas-Sanz F see Pastor

Fang, H.-P., see Cao

Ferrer, S., see Pascua

Feudel, U., Safonova, M.A., Kurths, J. \& Anishchenko, V.S., On the destruction of three-dimensional Tori

Fiedler, B., \& Turaev, D., Coalescence of reversible homoclinic orbits causes elliptic resonance

Finger, L., On the dynamics of coupled Josephson junction circuits

Forbes, L.K., see Sidhu

Fossas, E., see Batlle

Foster, E.A.D., Boundary crises arising from saddle-node bifurcations in periodically forced models

Franco, H., Wavelet analysis of a nonlinear oscillator transient during synchronization

Franz, M., see Huang

Freund, J., see Ebeling

Froeschle, C., see Celletti

Fussy, S., Grössing, G. \& Schwabl, H., Fractal evolution in deterministic and random models

Galias, Z., see Ogorzalek

Gardini, L., see Mira

Georgiou, I.T., \& Schwartz, I.B., The slow invariant manifold of a conservative Pendulum-oscillator system

Ghrist, R.W., \& Holmes, P.J., An ODE whose solutions contain all knots and links

Giaquinta, A., Boccaletti, S. \& Arecchi, F.T., Superexcitability induced spiral breakup in excitable systems

Gicquel, N., Bifurcation structure in a transmission system modelled by a two-dimensional endomorphism

Glass, K., Adachi, M. \& Mees, A., Noise tolerance of the AGOY control method for stabilizing chaotic systems

Glendinning, P., \& Sparrow, C., Shilnikov's saddle-node bifurcation

Gligoroski, D., Dimovski, D., Kocarev, L., Urumov, V. \& Chua, L.O., A method for encoding messages by time targeting of the trajectories of chaotic systems

Godivier, X., see Chapeau-Blondeau

Gómez-Gesteira, M., see de Castro

Gómez-Gesteira, M., see Muñuzuri

Gonchenko, S.V., Sten'kin, O.V. \& Turaev, D.V., Complexity of homoclinic bifurcations and $\Omega$-moduli

Gorodetski, A., \& Ilyashenko, Yu., Minimal and strange attractors Gouesbet, G., see Letellier

Grassi, G., see Brucoli

Gray, B.F., see Sidhu

Gray, R.A., \& Jalife, J., Spiral waves and the heart

Grebogi, C., see Poon

Grebogi, C., see Poon

Griffith, C.A. see Lapidus

Grössing, G., see Fussy

Guckenheimer, J., \& Malo, S., Computer-generated proofs of phase portraits for planar systems

Güémez, J., see Gutiérrez

Güémez, J., \& Matías, M.A., Synchronization in small assemblies of Van der Pol-Duffing chaotic oscillators

Guerra, J.M., see Pastor

Gutiérrez, J.M., Iglesias, A., Guiémez, J. \& Matías, M.A., Suppression of chaos through changes in the system variables through Poincaré and Lorenz return maps 
Harb, A.M., see Nayfeh

Hartbauer, M., see Windisch

Hilborn, R.C., \& Ding, M., Optimal reconstruction space for estimating correlation dimension

Hofer, E., Mohr, G., Jorge, A.C., Platzer, D. \& Schafferhofer, I., Is the discrete coupling of cardiac cells reflected in the wavefront of extracellular potentials? - An experimental approach

Hofer, E., see Muñuzuri

Holden, A.V., Poole, M.J. \& Tucker, J.V., An algorithmic model of the mammalian heart: Propagation, vulnerability, re-entry and fibrillation

Holden, A.V., see Kremmydas

Holden, A.V., see Biktashev

Holmes, P.J., see Ghrist

Holmes, W.T., see Moon

Hsu, S.-B., see Chen

$\mathrm{Hu}$, J., see Atela

Huang, A., see Pivka

Huang, A.S., Pivka, L., Wu, C.W. \& Franz, M., Chua's equation with cubic nonlinearity

Huang, G., Lou, S.Y. \& Velarde, M.G., Gap solitons, resonant kinks, and intrinsic localized modes in parametrically excited diatomic lattices

Huang, Y.S., \& Aronson, D.G., Discrete rotating wave solutions for system of globally coupled Josephson junctions

Huerta, R., A finite automata model of spiking-bursting neurons

Hwang, C., see Chen

Hynne, F., see Wang

Iglesias, A., see Gutiérrez

Ilyashenko, Yu., see Gorodetski

Jalife, J., see Gray

Jiménez-Montaño, M.A., see Storella

Johnson, M.A., see Moon

Jorge, A.C., see Hofer

Kaiser, F., see Sauer

Kapitaniak, T., see Sekieta

Kapitaniak, T., Sekieta, M. \& Ogorzalek, M., Monotone synchronization of chaos

Kapitaniak, T., \& Chua, L.O., Locally-intermingled basins of attraction in coupled Chua's circuits

Kaplan, B.Z., see Sarafian

Kappos, E., The Conley Index and global bifurcations. II. Illustrative applications

Kaschenko, S.A., Normalization in the systems with small diffusion

Kazantsev, V.B., see Nekorkin

Kazantsev, V.B., see Nekorkin

Kim, Y., see Shin

Kinouchi, O., \& Tragtenberg, M.H.R., Modeling neurons by simple maps

Kiriki, S., The Palis and Takens' problem on the first homoclinic tangency inside the horseshoe

Klemm, M., \& Beckmann, P.E., The topology of basin boundaries in a class of three-dimensional dynamical systems

Klíč, A., \& Pokorný, P., On dynamical systems generated by two alternating vector fields

Knudsen, C., Topological winding numbers for period-doubling cascades

Kocarev, L., see Gligoroski

Kocarev, L., see Stojanovski

Kocarev, L., see Parlitz

Kokubu, H., Komuro, M. \& Oka, H., Multiple homoclinic bifurcations from orbit-flip. I. Successive homoclinic doublings

Kollmann, M., see Vrahatis
Koltsova, O.Yu., \& Lerman, L.M., Families of transverse Poincare homoclinic orbits in $2 \mathrm{~N}$-dimensional Hamiltonian systems close to the system with a loop to a saddle-center

991

833

Korolev, V.G., see Eleonsky

Kozlov, A.K., Shalfeev, V.D. \& Chua, L.O., Exact synchronization of mismatched chaotic systems

Kremmydas, G.P., Holden, A.V., Bezerianos, A. \& Bountis, T., Representation of sino-atrial node dynamics by circle maps

Krouskop, T.A., see Chiou-Tan

Kuminov, D.A., see Andreyev

Kurths, J., see Feudel

Kusch, I., see Markus

Kuznetsov, A.P., Kuznetsov, S.P., Sataev, I.R. \& Chua, L.O., Multiparameter criticality in Chua's circuit at period-doubling transition to chaos

Kuznetsov, A.S., see Shalfeev

Kuznetsov, S.P., see Kuznetsov

Kuznetsov, Yu.A., see Champneys

Kwun, S.-I., see Shin

Lacy, J.G., A simple piecewise-linear non-autonomous circuit with chaotic behavior

Lai, D., see Chen

Lakshmanan, M., see Lipton

Lansbury, A.N., see Arrowsmith

Lapidus, M.L., Neuberger, J.W., Renka, R.J. \& Griffith, C.A., Snowflake harmonics and computer graphics: Numerical computation of spectra on fractal drums

Le Van Quyen, M., Schuster, H.G. \& Varela, F.J., Fast dynamics coupling from slow neuronal oscillators

Lega, E., see Celletti

Lerman, L.M., see Koltsova

Letellier, C., Gouesbet, G. \& Rulkov, N.F., Topological analysis of chaos in equivariant electronic circuits

Leung, A.Y.T., \& Rajendran, S., Symmetry and bifurcation of a three-hinged rod

1799

Li, Q.-L., see Cao

Linsay, P.S., see Brailove

Lipton, J.M., \& Dabke, K.P., Softening the nonlinearity in Chua's circuit

Lipton, J.M., \& Dabke, K.P., Spread spectrum communications based on chaotic systems

Lipton, J.M., Dabke, K.P. \& Lakshmanan, M., Bispectral properties of the simplest dissipative nonautonomous chaotic circuit

Liu, Z., \& Zhu, Z., Strange nonchaotic attractors from quasiperiodically forced Ueda's circuit

Lohmann, M., \& Wenzelburger, J., A statistical method for detecting cycles in discrete dynamical systems

Lord, G.J., see Bai

Loskutov, A.Yu., Tereshko, V.M. \& Vasiliev, K.A., Stabilization of chaotic dynamics of one-dimensional maps by a cyclic parametric transformation

Lou, S.Y., see Huang

Lutzenberger, W., see Birbaumer 267

Magee, K.N., see Chiou-Tan 1389

Maistrenko, Y., see Mira 2299

Makarov, V.A., see Nekorkin 1845

Mallet-Paret, J., see Chow 1605

Malo, S., see Guckenheimer $\quad 889$

Mar, G., \& Denis, P.St., Real life 2077

Marino, I.P., see de Castro 1725

Markus, M., Kusch, I., Ribeiro, A. \& Almeida, P., Class IV behavior 1817 in cellular automata models of physical systems

Markus, M., see Muñuzuri 
Marques, F., see Meseguer

Maru, V.M., see Singh

Maru, V.M., see Singh

Matías, M.A., see Gutiérrez

Matías, M.A., see Guiémez

Matrosov, V.V., see Ponomarenko

Mayer-Kress, G., see Birbaumer

Mayer-Kress, G., see Mettin

Mazin, W., see Rasmussen

McGehee, R.P., \& Peckham, B.B., Arnold flames and resonance surface folds

Mees, A., see Glass

Mendes, E.M.A.M., see Aguirre

Meseguer, A., Marques, F. \& Sanchez, J., Feigenbaum's universality in a low-dimensional fluid model

Mettin, R., \& Mayer-Kress, G., Chaotic attractors from homotopic mixing of vector fields

Milanovíc, V., \& Zaghloul, M.E., Synchronization of chaotic neural networks and applications to communications

Millerioux, G., see Mira

Mira, C., Carcasses, J.-P., Millerioux, G. \& Gardini, L., Plane foliation of two-dimensional noninvertible maps

Mira, C., Rauzy, C., Maistrenko, Y. \& Sushko, I., Some properties of a two-dimensional piecewise-linear noninvertible map

Miyano, T., Time series analysis of complex dynamical behavior contaminated with observational noise

Moharir, P.S., see Singh

Moharir, P.S., see Singh

Mohr, G., see Hofer

Moiola, J.L., \& Chen, G., On the birth of multiple limit cycles in nonlinear systems

Moiola, J.L., Chiacchiarini, H.G. \& Desages, A.C., Bifurcations and Hopf degeneracies in nonlinear feedback systems with time delay

Mondragón C, R.J., see Arrowsmith

Moon, F.C., Johnson, M.A. \& Holmes, W.T., Controlling chaos in a two-well oscillator

Mosekilde, E., see Rasmussen

Moss, F., see Chiou-Tan

Müller, W., see Windisch

Muñuzuri, A.P., De Castro, M., Hofer, E., Pérez-Muñuzuri, V., Gómez-Gesteira, M. \& Pérez-Villar, V., Continuous conductive volume affects the propagation of signals in discrete systems

Muñuzuri, A.P., \& Markus, M., Cellular automaton model and measurements of autowave splitting

Narayaninsamy, T., On a class of fractional iterates

Nayfeh, A.H., Harb, A.M. \& Chin, C.-M., Bifurcations in a power system model

Nekorkin, V.I., Kazantsev, V.B. \& Chua, L.O., Chaotic attractors and waves in a one-dimensional array of modified Chua's circuits

Nekorkin, V.I., Makarov, V.A. \& Velarde, M.G., Spatial disorder and waves in a ring chain of bistable oscillators

Nekorkin, V.I., Kazantsev, V.B. \& Velarde, M.G., Travelling waves in a circular array of Chua's circuits

Nelson, M.R., see Chiou-Tan

Neuberger, J.W., see Lapidus

Nicolis, C., see Vannitsem

Nikolaev, E.V., see Biktashev

Ogorzalek, M., see Kapitaniak

Ogorzalek, M.J., Galias, Z., Dąbrowski, A. \& Dạbrowski, W., Wave propagation, pattern formation and memory effects in large arrays of interconnected chaotic circuits

Oka, H., see Kokubu
1587

383

759

1351

1761

2087

267

395

1077

315

1333

279

2571

1439

2031

383

759

1767

2587

661

437

1077

1389

1925

1829
Oketani, N., \& Ushio, T., Chaotic synchronization of globally coupled maps with an application in communication 2145

Olivar, G., see Batlle 2635

Ott, E., see Poon 1409

Ott, E., see Poon

Panas, A.I., see Dmitriev

Pandin, M., \& Didoné, G., Chaos in information processing: Simulation of a biological learning process by time evolution in an unsupervised neural network

Parlitz, U., see Stojanovski

Parlitz, U., \& Kocarev, L., Multichannel communication using autosynchronization

Pascua, P., Rubio, J.L., Viartola, A. \& Ferrer, S., Visualizing relative equilibria and bifurcations by painting Hamiltonians on personal computers

Pasemann, F., see Wennekers

Pasemann, F., see Stollenwerk

Paskota, M., On local control of chaos: The neighbourhood size

Pastor, I., Pérez-García, V.M., Encinas-Sanz, F. \& Guerra, J.M., Different and coexisting routes to chaos along a common bifurcation path in the Maxwell-Bloch equations

Peckham, B.B., see McGehee

Pei, L.-Q., see Chen

Pei, L.-Q., see Chen

Pelinovsky, D.E., \& Yakhno, V.G., Generation of collective-activity structures in a homogeneous neuron-like medium. I. Bifurcation analysis of static structures

Pelinovsky, D.E., \& Yakhno, V.G., Generation of collective-activity structures in a homogeneous neuron-like medium. II. Dynamics of propagating and pulsating structures

Pellegrini, L., see Possio

Péntek, A., Tél, T. \& Toroczkai, Z., Transient chaotic mixing in open hydrodynamical flows

Pereira, J.V., Bifurcational behavior of the state equation for an assembly of interacting resonators

Pérez, A.T., Chacón, R. \& Castellanos, A., Behavior of dynamical systems subjected to continuous and discontinuous forcing: Application to laminar chaotic mixing

Pérez-García, V.M., see Pastor

Pérez-Muñuzuri, V., see de Castro

Pérez-Muñuzuri, V., see Muñuzuri

Pérez-Villar, V., see de Castro

Pérez-Villar, V., see Muñuzuri

Piacquadio Losada, M., Planar tilings as fractal configurations

Pismen, L.M., Rubinstein, B.Y. \& Velarde, M.G., On automated derivation of amplitude equations in nonlinear bifurcation problems

Pivka, L., see Huang

Pivka, L., Wu, C.W. \& Huang, A., Lorenz equation and Chua's equation

Platzer, D., see Hofer

Plaza, F., \& Velarde, M.G., Avalanche-collapse mechanism: A model for excitability

Pokorný, P., see Klíč

Pokrovskii, A., see Diamond

Ponomarenko, V.P., \& Matrosov, V.V., Nonlinear dynamics of multistable Chua's circuits

Pontes, J., see Bragard

Pontes, J., Christov, C.I. \& Velarde, M.G., Numerical study of patterns and their evolution in finite geometries

Poole, M.J., see Holden

Poon, L., Campos, J., Ott, E. \& Grebogi, C., Wada basin boundaries in chaotic scattering

Poon, L., Campos, J., Ott, E. \& Grebogi, C., Wada basin boundaries in chaotic scattering (Errata)
251

851

203

2645

2145
435
409
51
851

2055

693

169

589

315

1595

2441

81 
Possio, C.T., \& Pellegrini, L., The influence of Hopf bifurcation degeneracies on the dynamic behavior of a non-ideal pi controlled CSTR

Raab, V., Induced hysteresis-free transitions in a bistable system

Rajendran, S., see Leung

Ranjan, R., see Casaleggio

Rapp, P.E., see Storella

Rasmussen, K.E., Mazin, W., Mosekilde, E., Dewel, G. \& Borckmans, P., Wave-splitting in the bistable Gray-Scott model

Rateitschak, K., see Ebeling

Rau, H., see Birbaumer

Rauzy, C., see Mira

Rega, G., \& Salvatori, A., Bifurcation structure at 1/3-subharmonic resonance in an asymmetric nonlinear elastic oscillator

Renka, R.J., see Lapidus

Ribeiro, A., see Markus

Robinson, L.R., see Chiou-Tan

Rubinstein, B.Y., see Pismen

Rubio, J.L., see Pascua

Rulkov, N.F., see Letellier

Safonova, M.A., see Feudel

Salvatori, A., see Rega

Sanchez, J., see Meseguer

Sandstede, B., see Champneys

Sarafian, G., \& Kaplan, B.Z., On the question of dissipation and parametric excitation in chaotic nonautonomous resonators

Sataev, E.A., Convergence of invariant measures for degenerating sequences of hyperbolic mappings with singularities

Sataev, E.A., Convergence of invariant measures for degenerating sequences of hyperbolic mappings with singularities (Errata)

Sataev, I.R., see Kuznetsov

Sauer, M., \& Kaiser, F., Crisis-induced intermittency in a spatially extended nonlinear optical system

Sbitnev, V.I., Clustering in a coupled map lattice

Schaffer, P., see Windisch

Schafferhofer, I., see Hofer

Schenk-Hoppe, K.R., see Arnold

Schuster, H.G., see Le Van Quyen

Schütz, P., \& Bode, M., Discrete coupling and propagating signals

Schwabl, H., see Fussy

Schwartz, I.B., see Carr

Schwartz, I.B., see Georgiou

Sekieta, M., \& Kapitaniak, T., Practical synchronization of chaos via nonlinear feedback scheme

Sekieta, M., see Kapitaniak

Sendhoff, B., see Wienholt

Setti, G., see Crounse

Shalfeev, V.D., \& Kuznetsov, A.S., Controlling pattern formation in a CNN of Chua's circuits

Shalfeev, V.D., see Kozlov

Shashkov, M.V., \& Turaev, D.V., On the complex bifurcation set for a system with simple dynamics

Shaw, S.W., see Chen

Shen, W.-X., see Afraimovich

Shi, Y., see Storella

Shin, J.C., Kwun, S.-I. \& Kim, Y., Experimental tracking of unstable orbits in driven diode resonator by weak coupling

Short, K.M., Unmasking a modulated chaotic communications scheme

Shub, M., see Blum

Sidhu, H.S., Forbes, L.K. \& Gray, B.F., Periodically forced hydrocarbon oxidation reaction in a CSTR

Sinai, Ya.G., A remark concerning the thermodynamical limit of the Lyapunov spectrum
Singh, R., Moharir, P.S. \& Maru, V.M., Compound chaos 383

Singh, R., Moharir, P.S. \& Maru, V.M., Mutual and selfcompounding of chaotic systems

Smale, S., see Blum

Soliman, M.S., Sub-Critical flip bifurcations that may or may not result in period-doubling

Sørensen, P.G., see Wang

Spach, M.S., The stochastic nature of cardiac propagation due to the discrete cellular structure of the myocardium

Sparrow, C., see Glendinning

Spence, A., see Bai

Sri Namachchivaya, N., see Arnold

Stämpfle, M., Cellular automata and optimal path planning

Starkov, S.O., see Dmitriev

Starmer, C.F., \& Starobin, J., Spiral tip movement: The role of the action potential wavelength in polymorphic cardiac arrhythmias

Starobin, J., see Starmer

Sten'kin, O.V., see Gonchenko

Stojanovski, T., Kocarev, L. \& Parlitz, U., A simple method to reveal the parameters of the Lorenz system

Stollenwerk, N., \& Pasemann, F., Control strategies for chaotic neuromodules

Storella, R.J., Shi, Y., Wood, H.W., Jiménez-Montaño, M.A., Albano, A.M. \& Rapp, P.E., The variance and the algorithmic complexity of heart rate variability display different responses to anesthesia

Suárez, R., Alvarez-Ramirez, J. \& Aguirre, B., First Harmonic analysis of planar linear systems with single saturated feedback

Sushko, I., see Mira

Tél, T., see Péntek

Tereshko, V.M., see Loskutov

Thakor, N.V., see Casaleggio

Thiran, P., see Crounse

Tokuda, I., Tokunaga, R. \& Aihara, K., A simple geometrical structure underlying speech signals of the Japanese Vowel /al

Tokunaga, R., see Tokuda

Toroczkai, Z., see Péntek

Tragtenberg, M.H.R., see Kinouchi

Tucker, J.V., see Holden

Tuel, S.S., see Chiou-Tan

Tuma, E., see Blázquez

Turaev, D., see Fiedler

Turaev, D., On dimension of non-local bifurcational problems

Turaev, D.V., see Shashkov

Turaev, D.V., see Gonchenko

Uchimura, K., The dynamical systems associated with Chebyshev polynomials in two variables

Urumov, V., see Gligoroski

Ushio, T., see Oketani

van Dooren, R., Bifurcations and chaos in a pendulum with circular support motion

van Vleck, E.S., see Chow

Vannitsem, S., \& Nicolis, C., Error growth dynamics in spatially extended systems

Varela, F.J., see Calenbuhr

Varela, F.J., see Le Van Quyen

Vasiliev, K.A., see Loskutov

Velarde, M.G., see Bragard

Velarde, M.G., see Huang

Velarde, M.G., see Nekorkin

Velarde, M.G., see Plaza

Velarde, M.G., see Pontes

Velarde, M.G., see Pismen

Velarde, M.G., see Nekorkin 
Vesin, J.-M., see Vibe

529

Viartola, A., see Pascua

Vibe, K., \& Vesin, J.-M., On chaos detection methods

Vrahatis, M.N., Bountis, T.C. \& Kollmann, M., Periodic orbits and invariant surfaces of $4 D$ nonlinear mappings

Wang, J., Hynne, F. \& Sørensen, P.G., Period-doubling geometry of the Belousov-Zhabotinsky reaction dynamics

Wennekers, T., \& Pasemann, F., Synchronous chaos in highdimensional modular neural networks

Wenzelburger, J., see Lohmann

Wienholt, W., \& Sendhoff, B., How to determine the redundancy of noisy chaotic time series

Windisch, H., Müller, W., Ahammer, H., Schaffer, P., Dapra, D. \& Hartbauer, M., Optical potential mapping helps to reveal discrete-natural-phenomena in cardiac muscle

Wolf, E.E., see Chen

Wood, H.W., see Storella

Wu, C.W., \& Chua, L.O., On a variation of the Huberman-Lumer adaptive scheme

Wu, C.W., see Chua

Wu, C.W., see Huang

Wu, C.W., see Pivka
Wu, C.W., Yang, T. \& Chua, L.O., On adaptive synchronization and control of nonlinear dynamical systems

Wu, C.W., see Andreyev

Wu, C.W., \& Chua, L.O., On the generality of the unfolded Chua's Circuit

Xie, Q.-X., \& Chen, G.-R., Synchronization stability analysis of the chaotic Rossler system

Yakhno, V.G., see Pelinovsky

Yakhno, V.G., see Pelinovsky

Yang, T., see Chua

Yang, T., \& Chua, L.O., Channel-independent chaotic secure communication

Yang, T., see $W u$

Yin, Y.-Z., Synchronization of chaos in a modified Chua's circuit using continuous control

Zaghloul, M.E., see Milanovíc

Zhang, H., Interactions between vortices and lesions and ectopic Foci in a CML model of cardiac tissue

Zhong, G.-Q., see Chua

Zhou, J., see Chen

1509

Zhu, Z., see Liu 